
\title{
28 Research Square \\ TB-diabetes comorbidity and associated factors in Maekel Zone, Eritrea: A cross sectional study.
}

\section{Zenawi Zeramariam Araia ( $\square$ zenhopncl@gmail.com )}

Ministry of Health Eritrea https://orcid.org/0000-0002-5756-438X

Amanuel Hadgu Mebrahtu

National TB and Leprosy Control Program, Ministry of Health

Adiam Ghebreyohanns Tewelde

Intergrated Diseases Surveillance and Response, Ministry of Health

\section{Araia Berhane Mesfun}

Communicable Diseases Control Division, Ministry of Health

\section{Randa Osma Saleh}

Asmara College of Health Sciences

Hagos Andom Tuumzghi

Ministry of Health, Maekel zone

\section{Research article}

Keywords: TB-DM, comorbidity, Eritrea, pulmonary TB, diabetes risk factors

Posted Date: December 9th, 2019

DOl: https://doi.org/10.21203/rs.2.18487/v1

License: (c) (i) This work is licensed under a Creative Commons Attribution 4.0 International License.

Read Full License 


\section{Abstract}

Background : In Eritrea, no study document the true burden of TB-diabetes comorbidity. However, diabetes has been constantly increasing with a prevalence of $3.4 \%$. Similarly, even though TB notification is showing a declining trend the prevalence is $123 / 100,000$ population. With an increasing diabetes trend, the country is at higher risk of rising TB-diabetes comorbidity which can further complicate TB care, control and prevention activities. Thus, this study was conducted to assess the prevalence of diabetes among pulmonary positive TB patients and its influencing factors in Maekel zone, Eritrea.

Methods: This study was a cross sectional study conducted in all (11) TB diagnostic and treatment sites of Maekel zone. All consecutive pulmonary positive TB cases who were registered during the period of 2015-2018 in the national TB treatment register and were eligible included in the analysis. Pretested data extraction tool was used to collect the required information. Data analysis was done by SPSS version 23. Simple frequencies, percentages and means were used to describe the data. Chi-square test and logistic regression analysis was also performed.

Results: A total of 490 pulmonary positive TB cases were eligible for the study and the overall prevalence of diabetes among pulmonary positive TB cases was $4.3 \%$. Univariate logistic regression showed that participants with an older age (45-90) were more likely to have TB-diabetes comorbidity (COR: 4.397[Cl: 1.675-11.545], $p<0.01$ ) than those aged 10-44 years old. In addition, subjects whose weight was $65 \mathrm{~kg}$ and above were more likely (COR: 7.647[Cl: 1.965-29.758], p<0.01) to have TB- diabetes comorbidity than those with lower body weight.

Conclusion: TB-diabetes comorbidity observed in this study is low but given the growing diabetes trend in the country, an integrated TB-DM services should be given regularly on full scale at all health facilities.

\section{Background}

Tuberculosis is still a cause of much public fear and stigma in many parts of the world accounting for millions of morbidity and mortality each year [1]. The burden of this global epidemic is bore not by the developed countries where this disease is regarded as a disease of the past but by low- and middleincome countries where ending TB is still a distant reality [1-5]. One of the key challenges in putting an end to TB is its changing landscape of care and prevention spurred by the continuous epidemiological and demographic transitions the world is currently witnessing. Such transitions include the increase in the burden of non-communicable diseases as well as the aging populations adding to the existing pool of risk factors and giving importance to previously overlooked comorbidities $[5,6]$.

A quite preventable but incurable public health concern currently in the rise is Diabetes Mellitus with an estimated 425 million cases in 2017, with numbers predicted to increase to 629 million by 2045[3].Countries found at the conjunction of TB and diabetes are faced with a double disease burden incurring catastrophic damages at the health system, family and individual level. Diabetes is a known risk factor for TB $[3,5,6,7,8]$ and it triples the risk of developing Tuberculosis with $15 \%$ of the global TB 
cases attributed to it $[9,10]$. According to a recent systematic review on the co-prevalence of Type 2 diabetes mellitus and TB, high burdens of diabetes in TB patients were found in Mexico (36.1\%), China (30.8\%), India (44\%), Marshall Islands (45\%) and Malaysia (41.3\%) followed by Kyrgyzstan (18.7\%), Ethiopia and Tanzania (15\%)[11]. The excess cases of TB attributable to diabetes have transcended the records set by the HIV-TB coinfection. Nowadays, globally there are now more TB patients living with concomitant diabetes than patients with concomitant HIV infection [8]. This increasing prevalence in TBdiabetes comorbidity offsets the expected effect of the intensified TB control efforts and represents a threat to the decades of progress made against TB [12].

Previously developed evidence base suggests that the relationship between TB and diabetes goes beyond an increased risk of developing TB with both diseases complicating each other at many levels. TB diagnosis among diabetic people could be challenging and TB patients with overlapping diabetes may experience adverse TB treatment outcome. In addition, diabetes may also accelerate the emergence of drug-resistant TB among those receiving TB treatment [3]. Conversely, TB may increase existing insulin resistance, trigger the onset of diabetes in predisposed individuals and worsens glycemic control in existing diabetes cases. Moreover, TB and DM medications may undermine each other's effectiveness through drug-drug interaction contributing to failure of TB treatment and glycemic control [3]. Thus, this double burden leads to substantial strain on individuals, families, health systems, and society [12].

According to the report of international diabetes federation, Eritrea has a diabetes prevalence of $3.4 \%$ which is constantly increasing [13]. At the same time, even though TB notification is showing a declining trend, the prevalence is $123 / 100,000$ populations [14]. In response to these burdens, the country has adopted and implemented the provisional collaborative framework for the care and control of TB and diabetes which was launched by the WHO and the International Union against TB and Lung Diseases [9]. Among all TB cases registered in the 2018 in Maekel zone, 5.4\% of them were also diabetic patients. [15]. With an increasing diabetes trend, the country is at higher risk of rising TB-diabetes comorbidity which can further complicate TB care, control and prevention activities. Thus, this study was conducted to assess the prevalence of diabetes among pulmonary positive TB patients and identify factors related to TB-diabetes comorbidity in Maekel zone, Eritrea.

\section{Methods}

\section{Study design}

The study was a cross sectional in design.

\section{Study setting and participants}

The study subjects were pulmonary positive TB cases who were treated for TB during the period 20152018 at all (11) TB diagnostic and treatment sites of Maekel zone. The study was conducted from June to August 2019. These health facilities include three community hospitals and eight health centers in which majority of them were located inside Asmara city. TB diagnosis was made based on the standard 
diagnostic procedures as stated in the National TB Control Program Guidelines [16]. Thus, consecutive pulmonary positive TB patients diagnosed based on the standard diagnostic procedure by sputum smear microscopy for acid fast bacilli and or by Gene-x-pert were included in this study. Similarly, Diabetic status of all study cases was found from the record as a self-reported history of diabetes or fasting plasma glucose $\geq 126 \mathrm{mg} / \mathrm{dl}$ or casual plasma glucose concentration $\geq 200 \mathrm{mg} / \mathrm{dl}$ as Defined by WHO [17].

\section{Inclusion criteria}

All pulmonary positive TB cases who were registered during the period of 2015-2018 in the national TB treatment register were included for analysis.

\section{Exclusion criteria}

All pulmonary negative and extra pulmonary TB cases were not part of this study. In addition, pulmonary positive TB patients with unknown or undocumented (missing) diabetic status were also excluded.

\section{Sampling technique and sample size}

The sampling technique used in this study was census sampling in which a total of 490 consecutive pulmonary positive TB cases who meet the inclusion criteria were included in the final analysis.

\section{Instruments and data collection}

Pretested data extraction tool was used to collect the required information from the medical records (national TB treatment register, TB laboratory register and TB treatment cards) available at respective health facility. The data extraction tool was developed by the authors based on TB Laboratory register, TB treatment register and TB patient treatment card which are adopted from the standard recording tools recommended by WHO. The data extraction tool consisted two sections, with the first section containing information on socio demographic characteristics of patients such as age, sex and residence. Section two covers information on clinical variable such as type of patient, patient category, laboratory results (smear microscopy/Gene-X-pert), HIV status, diabetes status and treatment outcome.

\section{Data analysis}

Data cleaning was performed for the collected information, then it was coded, entered and analyzed by SPSS version 23. Subjects with unknown or missing diabetic status were excluded from the analysis. Simple frequencies, percentages and means were calculated to describe the data. Chi-square test has been done to test the statistical significance of association of different socio demographic and clinical variable to the probability TB-DM coinfection. Univariater logistic regression analysis was performed but multivariate logistic regression analysis was not done due to small number of TB-DM cases. In this study, $\mathrm{p} \leq 0.05$ was considered as statistically significant. 


\section{Results}

After excluding two ineligible cases, a total of 490 pulmonary positive TB patients treated for TB in Maekel zone at the 11 health facilities during the period of January 2015 up to December 2018 were included in the analysis. The overall prevalence of diabetes among the pulmonary positive TB cases was 4.3\%. Male participants dominated in both TB patients with diabetes $(66.67 \%)$ and those without diabetes (55.44\%). Among TB-diabetes cases $71.42 \%$ were 45 and above years old whereas in TB cases without diabetes $49.04 \%$ were aged $25-44$ years. Similarly, mean age among TB-diabetes cases was 51.04 years which is higher than the mean age of those without diabetes 40.60 years. The average body weight among TB-diabetes case was $53.4 \mathrm{~kg}$ but in subject with TB disease only, it was $46.8 \mathrm{~kg}$ (Table 1).Generally, almost three fourth (74.49\%) of the study subjects and specifically $85.71 \%$ of the TBdiabetes cases and $73.98 \%$ of TB cases without diabetes were from urban areas.

Depending on their history of TB treatment, majority $(85.71 \%, 89.34 \%)$ of the cases were new in both TB with diabetes and without diabetes respectively. Previous TB treatment was reported among few (4.29\%) TB-diabetes and in $10.66 \%$ of TB cases without diabetes. Among those previously treated cases only relapse were found among TB-diabetes but treatment after failure and after lost to follow up were reported only among TB cases without diabetes comorbidity.

According to patients' treatment category, $85.71 \%$ of TB-diabetes cases and $89.13 \%$ of TB cases without diabetes belonged to the first treatment category. The least number of cases were documented in category three in which only $2(0.43 \%)$ TB patients without diabetes coinfection and none of those with TB-diabetes coinfection fall in that category. The overall HIV positivity in this study was $10 \%$ in which comparable HIV positivity of $9.52 \%$ and $10.02 \%$ was found among TB-diabetes and without diabetes respectively.

More interestingly, $95.24 \%$ of TB-diabetes cases were cured and only 1 case (4.76\%) died. Similarly, among TB case without diabetes, $92.75 \%$ were cured, $3.41 \%$ failed treatment, and $1.71 \%$ died (Table 2 ).

\section{Regression analysis}

The study found that age and weight of subjects were significantly associated with the presence of TBdiabetes comorbidity among pulmonary positive TB cases. Age of participants was categorized in to two groups as $10-44$ and $45-90$. Thus, univariate logistic regression showed that participants with an older age (45-90) were more likely to have TB-diabetes comorbidity (COR: 4.397[Cl: 1.675-11.545], $p<0.01$ ) than those aged 10-44 years old. Similarly, weight was categorized as $25-44 \mathrm{~kg}, 45-64 \mathrm{~kg}$, and $\geq 65 \mathrm{~kg}$. The finding revealed that subjects whose weight was $65 \mathrm{~kg}$ and above were more likely (COR: 7.647[Cl:1.965-29.758], p<0.01) to have TB- diabetes comorbidity than those with lower body weight(Table 3).

\section{Discussion}


In the present study, overall prevalence of diabetes among the pulmonary positive TB cases was $4.3 \%$. This was consistent to previous study that reported a prevalence of $4.7 \%$ in Zambia [18]. Higher prevalence of TB-diabetes comorbidity was also reported from Ethiopia 8.5\% [19], Portugal 6\% [20], Mexico 29.63\% [21], Iran 24.3\% [22], and India 25.3\% [23]. The prevalence of diabetes among TB patients observed in this study is comparable to the occurrence of the disease in the general population. While the difference among countries could be due to the inherent difference in socio economic and demographic characteristics among their population and the existing burden of both diseases in general population.

Many studies showed that male TB patients were more likely to suffer from TB-diabetes comorbidity than female TB patients $[19,20,22,23,24]$. The current study had also a consistent finding in which male subjects were proportionally dominant in both TB-diabetes cases and those without diabetes. In contrary to this, a study in Zambia described that majority of the TB-diabetes cases were females [18]. Presence of the comorbidity predominantly among male cases could be related to the relatively higher probabilities of smoking and alcohol drinking among men in Eritrea in which these risk factors could have increased the occurrence of both disease in this particular group.

Among TB-diabetes cases nearly three fourth were 45 and above years old whereas in TB cases without diabetes almost half of them were aged 25-44 years. Similarly, mean age among TB-diabetes cases was 51.04 years which is higher than the mean age of those without diabetes 40.60 years. Previous similar studies reported a consistent finding in which TB-diabetes cases were older than TB cases without diabetes and the difference was significant $[20,22,25]$. This could be described as age related diabetes because Type 2 diabetes frequently develops in older adults. This in turn could lead to the development of TB in infected individuals with the decreasing immunity due to diabetes. Opposing findings were also reported from Zambia where the mean age of participants was 33 and 37 years for TB-diabetes cases and those without diabetes respectively. None of the participants with coexisting TB-diabetes were above 50 years of age [18].

The present study failed to report BMI (body mass index) due to unavailability of height record for all patients. Thus, our result could not be compared to available literatures that reported $\mathrm{MBI}$ and should be interpreted carefully. Higher mean weight $(53.4 \mathrm{~kg})$ was observed among TB-diabetes cases in comparison to the TB cases without diabetes $(46.8 \mathrm{~kg})$. A likely description for effects of weight in relation to TB and diabetes has been documented in which higher risk of TB is detected in undernourished or underweight individuals and this risk decreases as individuals gain weight [12]. According to a study in Zambia, patients with coexisting TB-diabetes were more likely to have a normal or low BMI [19]. Likewise, a study from India reported lower mean BMI of TB-diabetes than those TB cases without diabetes [26]. Another study from UK found comparable BMI among TB-diabetes and those without diabetes [25]. Significant higher BMI in TB-diabetes patients was also reported [24, 27] that describes excessive weight gain predisposes to diabetes which is another independent risk factor for TB $[3,24]$. People with diabetes could have been over weight and may remain in over weight status after being affected by TB. On the other hand, there could be weight loss among diabetes cases due to TB that is infection induced hyperglycemia. 
Residential difference among study subjects had been related to varying occurrence of these diseases. Consequently, majority of the TB-diabetes cases and almost three fourth of TB cases without diabetes in the current study were residents of an urban areas. Earlier studies showed higher prevalence of diabetes among TB patients who were urban residents $[19,26]$. In contrast, a study in Northern India reported a higher number of study participants with TB-diabetes were living in rural areas [28]. The reason for higher TB-diabetes comorbidity among urban residents could be linked to the sedentary life style such as physical inactivity and unhealthy dietary intake. On the other hand there could be under diagnosis of these diseases in rural populations in relation to limited healthcare access or delay in healthcare seeking.

Depending on TB treatment history and category, majority of the cases were new case and in first treatment category in both TB-diabetes and without diabetes respectively. Previous TB treatment was reported among few (4.29\%) TB-diabetes and in $10.66 \%$ of TB cases without diabetes. Among those previously treated cases only relapse were found among TB-diabetes but treatment after failure and lost to follow up were reported only among TB cases without diabetes comorbidity. In agreement to the present study results, a study from UK reported that almost all cases of TB-diabetes and those without diabetes were new TB case and only less than $2 \%$ of them were previously treated [25]. Nevertheless, relatively higher number of TB-diabetes in category two with comparable figures among those TB cases without diabetes was reported previously $[24,26]$. This could be due to the fact that Eritrea has an excellent TB cases management and follow up reflected by very high TB treatment success rate which could have minimized the possibility of retreatment of TB cases.

Another health-related characteristic investigated by the study was HIV-infection. The overall HIV positivity in this study was $10 \%$ in which comparable HIV positivity was found among TB-diabetes and without diabetes. Studies in Zambia and Ethiopia had higher HIV positivity rate among TB-diabetes case $[18,19,22]$. A study in Portugal reported discrepant results with absence of HIV infection being a factor significantly associated with diabetes among TB patients [20]. HIV positivity rate found in this study was almost double of the prevalence of HIV in TB and almost 10 times higher than the prevalence in the general population. This could be due related to the study setting (Maekel zone) which may contain the highest number of HIV cases in the country.

In the current study, univariate logistic regression showed that participants with an older age (45-90) were more likely to have TB-diabetes comorbidity (COR: 4.397[Cl: 1.675-11.545], p < 0.01) than those aged 10-44 years old. In addition, subjects whose weight was $65 \mathrm{~kg}$ and above were more likely (COR: 7.647[Cl: 1.965-29.758], $\mathrm{p}<0.01$ ) to have TB- diabetes comorbidity than those with lower body weight. It's expected that at an older age in the presence of immunity compromising infection people could be more prone to develop TB disease leading to comorbidity.

Prior study revealed that TB patients aged 41and above had higher odds of having diabetes than younger patients [23]. Other literature also noted increasing age, family history of diabetes, sedentary occupation, body mass index, type of TB, category of TB, and smoking were significantly associated $[24,26]$. 


\section{Conclusions}

TB-diabetes comorbidity observed in this study is low but could not be ignored as increasing diabetes trend has been noticed in the country. Age $\geq 45$ years and body weight of $65 \mathrm{~kg}$ and above were significantly associated to TB-diabetes comorbidity among pulmonary positive TB cases. Early TB screening for diabetic patients and diabetes testing for TB patients should be continued regularly on full scale at health facilities.

\section{Limitations of the study}

Data for this study was collected from medical records in TB diagnostic and treatment sites. Thus, some information may be missing in which it hindered analysis of some important variable like BMI. In addition, there were few TB cases with diabetes and thus multivariate logistic regression was not performed.

\section{List Of Abbreviations}

BMl- body mass index

COR- crude odds ratio

DM- diabetes mellitus

HIV- human immune deficiency virus

IUATLD - international union against TB and lung disease

$\mathrm{MOH}$ - ministry of health

TB- tuberculosis

WHO- world health organization

\section{Declarations}

\section{Ethics approval and consent to participate}

The study was approved by the research clearance committee of the Research department of the Ministry of Health, Maekel branch. The heads of the health facilities which were included in the study were informed on the objectives of the study and were provided with written consent prior to data collection. Individual consent from study participants was not required due to data collection nature of the study. The data was collected anonymously and did not include any personal identifiers.

\section{Consent for publication}


Not applicable

\section{Availability of data and materials}

The data set analyzed in this study is available from the corresponding author on reasonable request.

\section{Competing interests}

Not applicable

\section{Funding}

Not applicable

\section{Authors' contribution}

$\mathrm{ZZ}$ conceived the idea, designed the study, analyzed the data and wrote the manuscript. $\mathrm{AH}$ and $\mathrm{HA}$ participated in data collection and entry. AY and RO prepared literature review and draft proposal. $A B$ critically assessed the manuscript. All authors have reviewed and read the final manuscript.

\section{Acknowledgments}

The authors would like to thank all TB focal persons in Maekel zone for their cooperation during the data collection time.

\section{References}

1. World Health Organization. Global tuberculosis report 2018. Licence: CC BY-NC-SA 3.0 IGO.

2. Seung Heon Lee. Tuberculosis Infection and Latent Tuberculosis. Tuberc Respir Dis 2016; 79:201206. org/10.4046/trd.2016.79.4.201

3. Lin Y, Harries A D, Kumar A M V, Critchley J A, van Crevel R, Owiti P, Dlodlo R A, Dejgaard A. Management of diabetes mellitus-tuberculosis: a guide to the essential practice. $1^{\text {st }}$ Paris, France: IUATLD, 2019.

4. World Health Organization. Treatment of tuberculosis Guidelines. 4th ed. WHO/HTM/TB/2009.420

5. D. Harries, S. Satyanarayana, A. M. V. Kumar, S. B. Nagaraja, P. Isaakidis, S. Malhotra, S.Achanta, B. Naik, N. Wilson, R. Zachariah, K. Lönnroth, A. Kapur. Epidemiology and interaction of diabetes mellitus and tuberculosis and challenges for care: a review. Public Health Action. 2013; 3(1). doi.org/10.5588/pha.13.0024

6. Blanca I. Restrepo. Microbiol Spectr. Diabetes and tuberculosis. 2016; 4(6): Doi:10.1128/microbiolspec.TNMI7-0023-2016.

7. Dooley KE, Chaisson RE. Tuberculosis and diabetes mellitus: convergence of two epidemics. The Lancet Infectious Diseases 2009; 9: 737-46.11. 
8. Girardi E, Shepisi MS, Goletti D, Bates M, Mwaba P, Yeboah-Manu D, Ntoumi F, Palmieri F, Maeurer M, Zumla A, Ippolito $G$. The global dynamics of diabetes and tuberculosis: the impact of migration and policy implication. International Journal of Infectious Diseases 56 (2017) 45-53. doi.org/10.1016/j.ijid.2017.01.018

9. Collaborative framework for care and control of tuberculosis and diabetes. WHO and IUATLD.2011. WHO/HTM/TB/2011.15

10. The dual epidemic of TB and Diabetes. World Health Organization. 2016. Available at https://www.who.int/tb/publications/diabetes_tb.pdf

11. Hannah Stowe McMurry, Emily Mendenhall, Aravind Rajendrakumar, Lavanya Nambiar, Srinath Satyanarayana, Roopa Shivashankar. Co-prevalence of type 2 diabetes mellitus and tuberculosis in low-income and middle-income countries: A systematic review. Diabetes Metab Res Rev. 2019; 35:e3066. Doi.org/10.1002/dmrr.3066.

12. Lönnroth $K$, Roglic $G$, Harries AD. Improving tuberculosis prevention and care through addressing the global diabetes epidemic: from evidence to policy and practice. Lancet Diabetes Endocrinol. 2014; 2: 730-39

13. World Health Organization. Diabetes country profiles, 2016. http://origin.who.int/diabetes/countryprofiles/eri_en.pdf accessed on 7/8/2019

14. World Health Organization. (2017). Global tuberculosis report. Country profile. WHO/HTM/TB/2071.23.Geneva, Switzerland.

15. National TB recording and reporting. 2018. Asmara, Eritrea.

16. National guideline for TB Control 2013-2017. Asmara, Eritrea.

17. Definition and diagnosis of diabetes mellitus and intermediate hyperglycemia. Report of a WHO/IDF Consultation. 2006. Geneva, Switzerland.

18. Fwoloshi S, Hachaambwa LM, Chiyeñu KO, Chirwa L, Hoffman TW, Ngalamika O, Bailey SL. Screening for Diabetes Mellitus among Tuberculosis Patients: Findings from a Study at a Tertiary Hospital in Lusaka, Zambia. Canadian Journal of Infectious Diseases and Medical Microbiology. 2018. doi.org/10.1155/2018/3524926.

19. Assefa Getachew, Solomon Mekonnen, Shiatye Alemu and Hanan yusuf. High magnitude of diabetes mellitus among Active Pulmonary Tuberculosis Patients in Ethiopia. British Journal of Medicine \& Medical Research. 2014; 4(3): 862-872

20. Cordeiro da Costa J, Oliveira O, Baía L, Gaio R, Correia-Neves M, Duarte R. Prevalence and factors associated with diabetes mellitus among tuberculosis patients: a nationwide cohort. Eur Respir $\mathrm{J}$ 2016; 48: 261-264. DOI: 10.1183/13993003.00112-2016.

21. Jiménez-Corona ME, Cruz-Hervert LP,García-García L, et al. Association of diabetes and tuberculosis: impact on treatment and post-treatment outcomes. Thorax 2013; 68:214-220. http://dx.doi.org/10.1136/thoraxjnl-2012-202976.

22. Seyed Mohammad Alavi, Mohammad Mehdi Khoshkhoy. Pulmonary tuberculosis and diabetes mellitus: Co-existence of both diseases in patients admitted in a teaching hospital in the southwest 
of Iran. Caspian J Intern Med 2012; 3(2): 421-424)

23. Pande T, Huddart S, XavierW, Kulavalli S, Chen T, PaiM, et al. (2018) Prevalence of diabetes mellitus amongst hospitalized tuberculosis patients at an Indian tertiary care center: A descriptive analysis. PLoS ONE 13(7): e0200838. https://doi.org/10.1371/journal.pone.0200838

24. Nagar V, Prasad P, Gour D, Singh AR, Pal DK. Screening for diabetes among tuberculosis patients registered under revised national tuberculosis control program, Bhopal, India. J Family Med Prim Care 2018; 7:1401-5. DOI:10.4103/jfmpc.jfmpc_14_18.

25. Ogbera AO, Kapur A, Abdur-Razzaq H, et al. Clinical profile of diabetes mellitus in tuberculosis. BMJ Open Diabetes Research and Care 2015;3:e000112.doi:10.1136/bmjdrc-2015000112.

26. Agarwal AK, Gupta G, Marskole P, Agarwal A. A study of the patients suffering from tuberculosis and tuberculosis-diabetes comorbidity in Revised National Tuberculosis Control Program Centers of Northern Madhya Pradesh, India. Indian J Endocr Metab 2017; 21:570-6. DOI:

10.4103/ijem.IJEM_89_17)

27. Alisjahbana B, van Crevel R, Sahiratmadja E, den Heijer M, Maya A, Istriana E, et al. Diabetes mellitus is strongly associated with tuberculosis in Indonesia. Int J Tuberc Lung Dis 2006;10:696-700

28. Surinder Pal Singh, Satinder Pal Singh, Jai Kishan, Sumeet Kaur, Shandhra Ramana. Association of tuberculosis and diabetes Mellitus: an analysis of 1000 consecutively admitted cases in a tertiary care hospital of North India. Pan African Medical Journal. 2016; 24:4.

Doi:10.11604/pamj.2016.24.4.8153

\section{Tables}

Table 1. Background characteristics of study participants

\begin{tabular}{l|c|c|c|}
\hline \multicolumn{3}{|c|}{ Pulmonary positive TB } & Total (\%) \\
\cline { 1 - 3 } Variable & Diabetic(n=21) & Non diabetic(n=469) & N=490 \\
\hline Sex (n=490) & & & \\
\hline Male & $14(66.67 \%)$ & $260(55.44 \%)$ & $274(55.92 \%)$ \\
\hline Female & $7(33.33 \%)$ & $209(44.56 \%)$ & $216(44.08 \%)$ \\
\hline Age range(n=490) & & & \\
\hline $10-44$ & $6(28.57 \%)$ & $299(63.75 \%)$ & $305(90.50 \%)$ \\
\hline 45-90 & $15(71.43 \%)$ & $170(36.25 \%)$ & $32(9.50 \%)$ \\
\hline Mean age(years) & 51.04 & 40.60 & 41.06 \\
\hline Body weight(Kg)(n=487) & & & $201(41.27 \%)$ \\
\hline 25-44 & $6(28.57 \%)$ & $195(41.85 \%)$ & $265(54.42 \%)$ \\
\hline 45-64 & $11(52.38 \%)$ & $254(54.50 \%)$ & $21(4.31 \%)$ \\
\hline 65 and above & $4(19.05 \%)$ & $17(3.65 \%)$ & $47.06 \%$ \\
\hline Mean body weight & 53.4 & 46.8 & $365(74.49 \%)$ \\
\hline Residence (n=490) & & & $125(25.51 \%)$ \\
\hline Urban & $18(85.71 \%)$ & $347(73.98 \%)$ & \\
\hline Rural & $3(14.29 \%)$ & $122(26.02 \%)$ & \\
\hline
\end{tabular}


Table 2. Clinical characteristics of study subjects

\begin{tabular}{l|c|c|c|}
\hline \multicolumn{4}{|c|}{ Clinical characteristics } \\
\hline Variable & \multicolumn{2}{|c|}{ Pulmonary positive TB } & Total $\%)$ \\
\hline & Diabetic $(\mathrm{n}=21)$ & Non diabetic $(\mathrm{n}=469)$ & $(\mathrm{n}=490)$ \\
\hline Type of TB patient & & & \\
\hline New & $18(85.71 \%)$ & $419(89.34 \%)$ & $437(89.18 \%)$ \\
\hline Previously treated & $3(14.29 \%)$ & $50(10.66 \%)$ & $53(10.82 \%)$ \\
\hline Patient category & & & \\
\hline I & $18(85.71 \%)$ & $418(89.13)$ & $436(88.97 \%)$ \\
\hline II & $3(4.29 \%)$ & $49(10.450$ & $52(10.62 \%)$ \\
\hline III & $0(0.00 \%)$ & $2(0.43)$ & $2(0.41 \%)$ \\
\hline HIV test result & & & $441(90.00 \%)$ \\
\hline Negative & $19(90.48 \%)$ & $422(89.98 \%)$ & $49(10.00 \%)$ \\
\hline Positive & $2(9.52 \%)$ & $47(10.02 \%)$ & \\
\hline Cured & & & $455(92.86 \%)$ \\
\hline Treatment completed & $20(0.00 \%)$ & $2(0.43 \%)$ & $2(0.41 \%)$ \\
\hline Failed & $0(0.00 \%)$ & $16(3.41 \%)$ & $16(3.36 \%)$ \\
\hline Died & $1(4.76 \%)$ & $8(1.71 \%)$ & $9(1.84 \%)$ \\
\hline Not evaluated & $0(0.00 \%)$ & $4(0.85 \%)$ & $4(0.82 \% 0$ \\
\hline
\end{tabular}

Table 3. Regression analysis 


\begin{tabular}{|c|c|c|c|c|}
\hline \multirow[b]{2}{*}{ Variable } & \multicolumn{2}{|c|}{ Pulmonary positive TB } & \multirow[t]{2}{*}{ COR[95\%CI] } & \multirow{2}{*}{$\begin{array}{l}\mathrm{p}- \\
\text { value }\end{array}$} \\
\hline & Diabetic $(n=21)$ & $\begin{array}{l}\text { Non } \\
\text { diabetic }(n=469)\end{array}$ & & \\
\hline \multicolumn{5}{|l|}{ Sex $(n=490)$} \\
\hline Male & $14(5.11 \%)$ & $260(94.89 \%)$ & $\mathrm{r}$ & \\
\hline Female & $7(3.24 \%)$ & $209(96.76 \%)$ & $0.622[.247-1.569]$ & 0.351 \\
\hline \multicolumn{5}{|l|}{ Age range $(n=490)$} \\
\hline $10-44$ & $6(1.97 \%)$ & $299(98.03)$ & $\mathrm{r}$ & \\
\hline $45-90$ & $15(8.11 \%)$ & $170(91.89 \%)$ & $4.397[1.675-11.545]$ & 0.003 \\
\hline \multicolumn{5}{|l|}{$\begin{array}{l}\text { Body weight(Kg) } \\
(\mathrm{n}=487)\end{array}$} \\
\hline $25-44$ & $6(2.99 \%)$ & 195(97.01\%) & $\mathrm{r}$ & \\
\hline $45-64$ & $11(4.15 \%)$ & $254(95.85 \%)$ & $1.407[0.512-3.872]$ & 0.508 \\
\hline 65 and above & $4(19.05 \%)$ & $17(80.95 \%)$ & $7.647[1.965-29.758]$ & 0.003 \\
\hline \multicolumn{5}{|l|}{ Residence $(n=490)$} \\
\hline Urban & $18(4.93 \%)$ & $347(95.07 \%)$ & $\mathrm{r}$ & \\
\hline Rural & $3(2.4 \%)$ & $122(97.6 \%)$ & $0.05[0.137-1.637]$ & 0.238 \\
\hline \multicolumn{5}{|l|}{ Type of TB patient } \\
\hline New & $18(4.12 \%)$ & $419(95.88 \%)$ & $\mathrm{r}$ & \\
\hline Previously treated & $3(5.66 \%)$ & $50(94.34 \%)$ & $1.397[0.397-4.909]$ & 0.602 \\
\hline \multicolumn{5}{|l|}{ Patient category } \\
\hline I & $18(4.13 \%)$ & $418(95.87 \%)$ & $\mathrm{r}$ & \\
\hline II & $3(5.77 \%)$ & $49(94.23 \%)$ & $1.422[0.404-5.000]$ & 0.583 \\
\hline III & $0(0.00 \%)$ & $2(100 \%)$ & $0.000[.000-]$ & 0.999 \\
\hline \multicolumn{5}{|l|}{ HIV test result } \\
\hline Negative & $19(4.30 \%)$ & $422(95.70 \%)$ & $\mathrm{r}$ & \\
\hline Positive & $2(4.08 \%)$ & $47(95.92 \%)$ & $\begin{array}{l}0.945[0.213- \\
4.185]\end{array}$ & 0.941 \\
\hline
\end{tabular}

